\title{
The Effect of Bilayer Graphene Nanoribbon Geometry on Schottky-Barrier Diode Performance
}

\author{
Meisam Rahmani, ${ }^{1}$ Razali Ismail, ${ }^{1}$ Mohammad Taghi Ahmadi, ${ }^{1,2}$ Mohammad Javad Kiani, ${ }^{3}$ \\ Mehdi Saeidmanesh, ${ }^{1}$ F. A. Hediyeh Karimi, ${ }^{4}$ Elnaz Akbari, ${ }^{4}$ and Komeil Rahmani ${ }^{5}$ \\ ${ }^{1}$ Faculty of Electrical Engineering, Universiti Teknologi Malaysia, 81310 Skudai, Johor Bahru, Malaysia \\ ${ }^{2}$ Nanotechnology Research Center Nanoelectronic Group, Physics Department, Urmia University, Urmia 57147, Iran \\ ${ }^{3}$ Department of Electrical Engineering, Islamic Azad University, Yasooj Branch, Yasooj 63614, Iran \\ ${ }^{4}$ Centre for Artificial Intelligence and Robotics (CAIRO), UTM, 81310 Skudai, Johor Bahru, Malaysia \\ ${ }^{5}$ Department of Electrical, Computer and Biomedical Engineering, Islamic Azad University, Qazvin Branch, Qazvin 34185-1416, Iran
}

Correspondence should be addressed to Razali Ismail; razali@fke.utm.my

Received 21 August 2013; Revised 11 October 2013; Accepted 17 October 2013

Academic Editor: Munawar A. Riyadi

Copyright (C) 2013 Meisam Rahmani et al. This is an open access article distributed under the Creative Commons Attribution License, which permits unrestricted use, distribution, and reproduction in any medium, provided the original work is properly cited.

\begin{abstract}
Bilayer graphene nanoribbon is a promising material with outstanding physical and electrical properties that offers a wide range of opportunities for advanced applications in future nanoelectronics. In this study, the application of bilayer graphene nanoribbon in schottky-barrier diode is explored due to its different stacking arrangements. In other words, bilayer graphene nanoribbon schottkybarrier diode is proposed as a result of contact between a semiconductor (AB stacking) and metal (AA stacking) layers. To this end, an analytical model joint with numerical solution of carrier concentration for bilayer graphene nanoribbon in the degenerate and nondegenerate regimes is presented. Moreover, to determine the proposed diode performance, the carrier concentration model is adopted to derive the current-voltage characteristic of the device. The simulated results indicate a strong bilayer graphene nanoribbon geometry and temperature dependence of current-voltage characteristic showing that the forward current of the diode rises by increasing of width. In addition, the lower value of turn-on voltage appears as the more temperature increases. Finally, comparative study indicates that the proposed diode has a better performance compared to the silicon schottky diode, graphene nanoribbon homo-junction contact, and graphene-silicon schottky diode in terms of electrical parameters such as turn-on voltage and forward current.
\end{abstract}

\section{Introduction}

Graphene nanoribbon (GNR) has attracted much attention of researchers for nanoelectronic applications because of its uniqueness electronic characteristics such as linear energy dispersion and width-tunable energy band gap [1-5]. Due to quantum confinement effect, GNR with width and thickness less than De-Broglie wave length $\left(\lambda_{D}=10 \mathrm{~nm}\right)$ can be assumed as a one-dimensional (1D) material [6]. GNR as an unwrapped carbon nanotube (CNT) is illustrated in Figure 1. It is notable that the properties of GNR are similar to CNT, but the planar structure of GNR guarantees better rectification current-voltage characteristic due to more accessible contact compared to other carbon-based materials [6]. Bilayer graphene nanoribbon (BGN) with unique physical and electrical properties has been incorporated in different nanoscale devices such as field effect transistors (FETs), tunnel transistors, and schottky diodes [7-11]. The BGN schottky-barrier diode has turned out to be of great interest holding the fact that it presents better performance compared to conventional semiconductor $p-n$ junction contacts [12]. In this paper, analytical modeling of BGN schottky-barrier diode is presented in which the effect of BGN geometry and temperature on the proposed diode performance is investigated.

\section{Schottky-Barrier Contact}

Schottky-barrier diode is a nonideal contact between a semiconductor and a metal. Rectification of alternating current is 


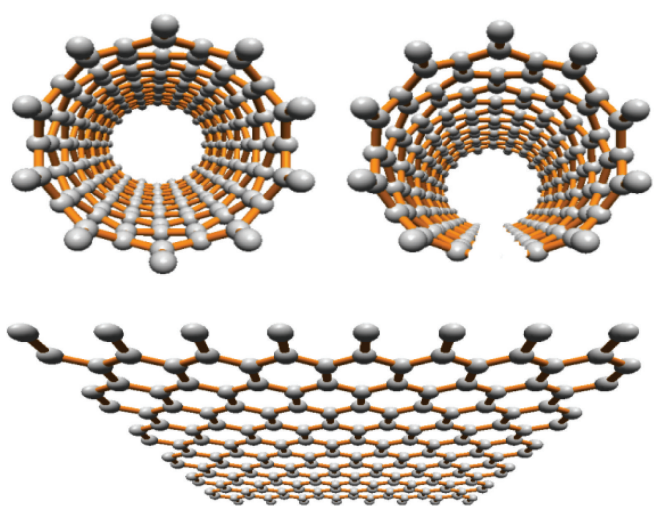

FIGURE 1: Graphene nanoribbon as a promising material for schottky contact.

a main relevant characteristic of the schottky-barrier diode [2]. The thermionic emission theory is known as the most important step in rectifying the contact with an $n$-type semiconductor throughout transport of electrons on top of the anticipated barrier [13]. Schottky-barrier diode based on GNR as a powerful device can be applied in a wide range of nanoelectronic applications because of its special advantages such as low injunction capacitance, low turn-on voltage, fast recovery time, and high operating frequencies $[13,14$, 16-18]. Also, RF signal rectification/detection, mixing, and imaging have been found to be some of its wide applications $[13,14,16-18]$. The different stacking arrangements of BGN $(\mathrm{AA}$ and $\mathrm{AB})$ in interlayer coupling between the top and bottom layers are weaker than those of interlayer coupling within layers leading to an electrical conduction $[14,19]$. The electrical conduction through the layers is exposed by the carrier hopping between the $\pi$ orbits, with the van der Waals forces contributing to the coupling interaction among the layers' structure [19]. The transport conduction channels are essentially generated by passing through the layers of BGN rather than between those layers [19]. As shown in Figure 2, the different configuration of $\mathrm{AB}$ stacking compared to $\mathrm{AA}$ is because of the shift in the distance of lattice in the armchair edges. Apparently, BGN with AB stacking is modeled in form of two honeycomb lattices with pairs of in-equivalent sites as $\left(A_{1}, B_{1}\right)$ and $\left(B_{2}, A_{2}\right)$ which are located in the top and bottom layers, respectively. However, in AA-stacked BGN, the pairs of in-equivalent sites $\left(A_{1}, B_{1}\right)$ and $\left(A_{2}, B_{2}\right)$ are located in the top and bottom layers, respectively. It is noteworthy that in $\mathrm{BGN}$ the carriers can move only in the $x$-direction. However, the carriers are confined to the $y$ - and $z$-Cartesian directions being less than the De-Broglie wave length [14, 19].

Different stacking sequences of BGN show metallic and semiconducting (with a band gap of $0.02 \mathrm{eV}$ ) properties, respectively [20-22]. This means that, by engineering of BGN different arrangements, the schottky-barrier contact can be designed as depicted in Figure 3.

\section{Proposed Model}

The energy dispersion relation of BGN in the attendance of a perpendicular electric field is obtained by the tight-binding technique. In this method, a self-consistent Hartree approximation can be utilized to estimate the induced charges on the different layers of BGN. This is because, as the Fermi surface of the intrinsic BGN communicates to the K-points, the tightbinding method is a suitable technique for the low energy excitations [23]. In addition, the tight-binding method with a single $p_{z}$ orbital per atom includes coupling parameters between the neighbor sites within BGN layers. In fact, the $p_{z}$ orbitals are considered to study the behavior of electrons around the Fermi level. The different $\mathrm{sp}^{2}$ orbitals that are lower than the $p_{z}$ orbital in energy will have much more overlap with the orbitals of the same symmetry on adjacent atoms [23]. Furthermore, the interaction between the $p_{z}$ orbitals of adjacent atoms is small, which results in bonding and antibonding orbitals close to the Fermi level. Therefore, the resulting bonding and antibonding molecular orbitals will recline below and above the Fermi level, respectively [23]. It is notable that the bonding and antibonding combinations are equal and communicate to orbitals which are limited to one of the two sublattices. This means that these orbitals are non-bonding in the nearest-neighbor approximation, so their relative energy is zero [23]. For the proposed $1 D$ BGN schottky-barrier diode, the tight-binding technique is adopted in order to calculate the energy band structure of BGN with AB stacking [24]:

$$
E_{k}(V)= \pm \sqrt{\epsilon_{k}^{2}+\frac{t_{\perp}^{2}}{2}+\frac{V^{2}}{4} \pm \sqrt{\left(t_{\perp}^{2}+V^{2}\right) \epsilon_{k}^{2}+\frac{t_{\perp}^{4}}{4}}}
$$

where $t_{\perp}$ is the interlayer hopping energy, $V$ is the applied voltage, and $\varepsilon_{k}^{2}=\left(V^{4} / 4+V^{2} t_{\perp}^{2} / 2\right) /\left(V^{2}+t_{\perp}^{2}\right)$. Equation (1) can be exposed as

$$
E(k) \approx \Delta-\alpha k^{2}+\beta k^{4}
$$

where $\Delta=V / 2$ is the bias voltage, $k$ is the wave vector, $\alpha=\left(V / t_{\perp}^{2}\right) v_{F}^{2}, \beta=v_{F}^{4} / V t_{\perp}^{2}, v_{F}=3 t \times a_{c-c} / 2$ is the Fermi velocity, and $a_{c-c}=1.41 \bar{\AA}$ is the lattice-spacing [25-28]. Figure 4 illustrates the energy band structure of BGN near the Fermi level which is plotted based on (2).

Unbiased BGN $(V=0)$ indicates zero band gap; however, energy dispersion of biased BGN by nonzero value of the applied voltage $(V)$ makes a gap between the conduction and valance bands. It is notable that the size of gap depends on the applied voltage and can be externally controlled by perpendicular electric field.

The density of states (DOS) as a fundamental factor shows the number of available states at each energy level that is occupied [29]. Hence DOS for BGN can be modeled as

$$
D(k)=\frac{1}{2 \pi\left(4 \beta k^{3}-2 \alpha k\right)} .
$$

Over energy band structure, carrier concentration can be calculated by integrating the distribution function as

$$
d n=\frac{1}{2 \pi\left(4 \beta k^{3}-2 \alpha k\right)\left(1+e^{\left(E-E_{F}\right) / k_{B} T}\right)} d E,
$$




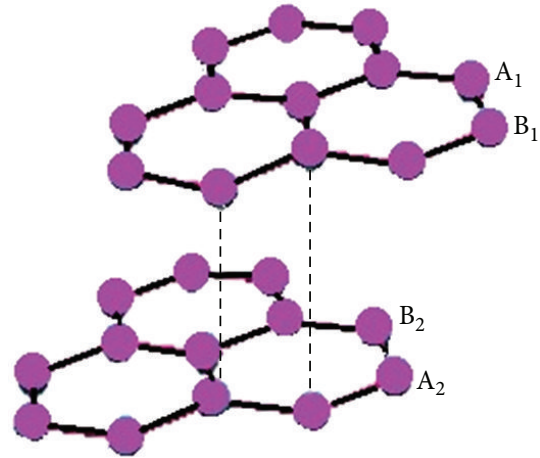

AB stacking

(a)

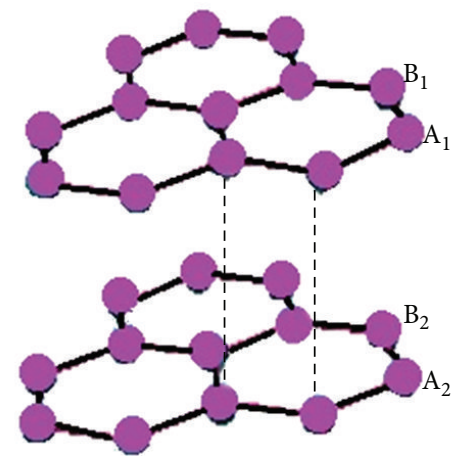

AA stacking

(b)

FIgURE 2: Configuration of BGN: (a) AB stacking and (b) AA stacking.

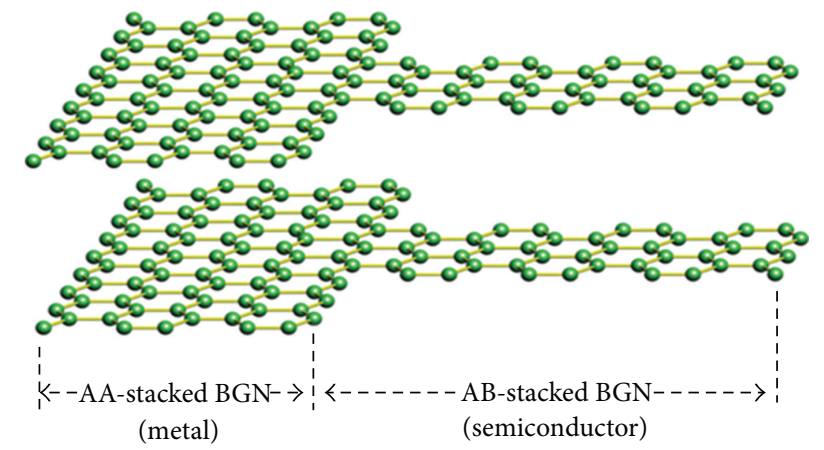

FIGURE 3: The proposed schematic of BGN schottky-barrier contact.

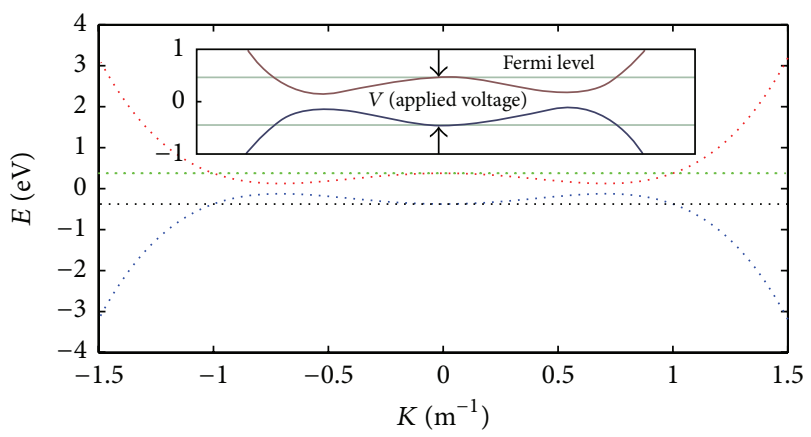

FIGURE 4: The energy band structure of BGN near the Fermi level.

where $k=\left[\left(\alpha \pm \sqrt{\alpha^{2}-4(E-\Delta) \beta}\right) / 2 \beta\right]^{1 / 2}$, which is achieved from the band energy equation (2). Considering the importance of carrier concentration, the proposed model of carrier concentration for BGN schottky-barrier diode in the degenerate and nondegenerate regimes is analytically studied. The band gap exhibits a nondegenerate approximation as the distance of Fermi level is more than $3 K_{B} T$ from either the conduction or valance bands [30]. In this region, because of high difference between $E$ and $E_{F}$, one (1) can be neglected in comparison with the exponential function as specified in
(4). Consequently, the model in the nondegenerate regime is carried out by the following equation as

$$
\begin{aligned}
& n_{0}^{\infty}\left(\left(e^{-\left(E-E_{f}\right) / k_{B} T} d E\right)\right. \\
& \quad \times\left(2 \pi \left[4 \beta\left(\frac{\alpha \pm \sqrt{\alpha^{2}-4(E-\Delta) \beta}}{2 \beta}\right)^{3 / 2}\right.\right. \\
& \left.\left.\left.\quad-2 \alpha\left(\frac{\alpha \pm \sqrt{\alpha^{2}-4(E-\Delta) \beta}}{2 \beta}\right)^{1 / 2}\right]\right)^{-1}\right) .
\end{aligned}
$$

Fermi level in the degenerate approximation is located less than $3 K_{B} T$ away from the conduction and valence bands or situated within a band [30]. In this case, we can neglect the $\exp \left(E-E_{F}\right)$ in comparison with (1) because the value of $E-E_{F}$ is very small. Therefore, the model of carrier concentration in the degenerate regime is proposed as

$$
\begin{aligned}
n_{\text {Degenerate }} & \\
\int_{0}^{\infty}(d E \times(2 \pi[ & 4 \beta\left(\frac{\alpha \pm \sqrt{\alpha^{2}-4(E-\Delta) \beta}}{2 \beta}\right)^{3 / 2} \\
& \left.\left.\left.-2 \alpha\left(\frac{\alpha \pm \sqrt{\alpha^{2}-4(E-\Delta) \beta}}{2 \beta}\right)^{1 / 2}\right]\right)^{-1}\right) .
\end{aligned}
$$

As shown in Figure 5, the proposed model of carrier concentration for BGN schottky-barrier diode is approximated by the nondegenerate limit, particularly in low value of the 


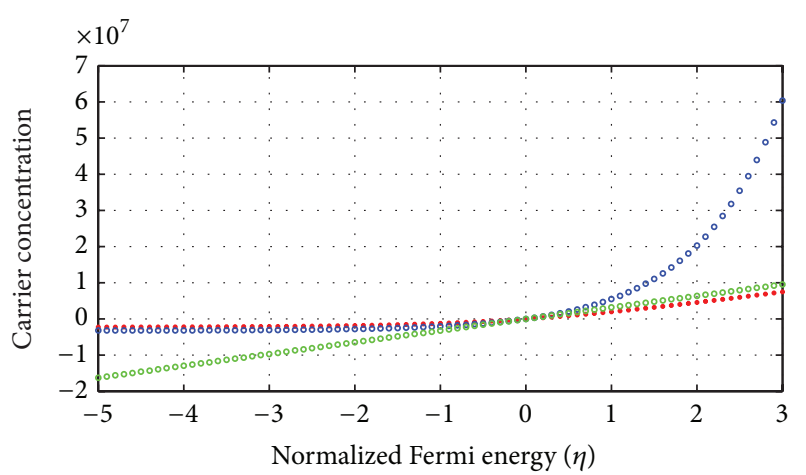

- Model

- Nondegenrate approximation

- Degenerate approximation

FIGURE 5: Carrier concentration of BGN in the degenerate and nondegenerate regimes.

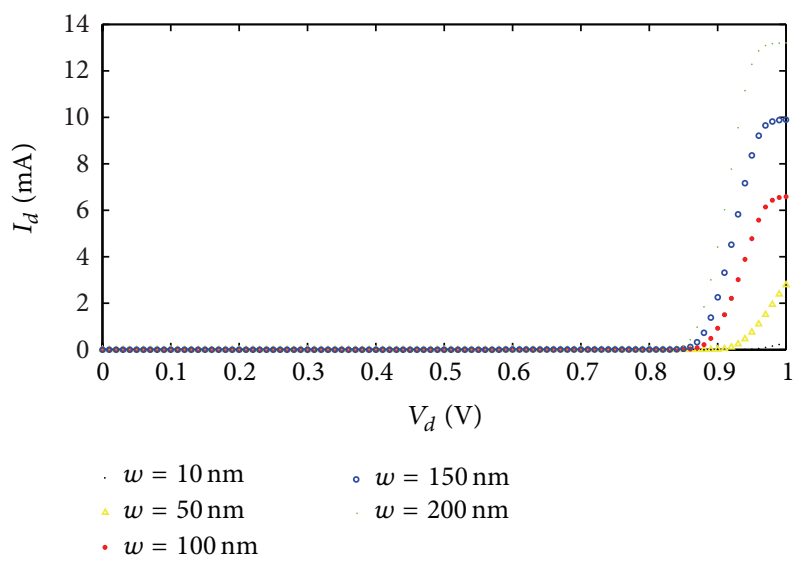

FIGURE 6: Rectification I-V characteristic of BGN schottky-barrier diode at different values of width $w(l=100 \mathrm{~nm})$.

normalized Fermi energy $\eta(\eta \leq 0.5)$, where $\left(\eta=\left(-E_{C}+\right.\right.$ $\left.\left.E_{f}\right) / k_{B} T\right)$. On the other hand, the model can be approximated by the degenerate limit, specifically in high value of $\eta(\eta>$ $0.5)$.

In the structure of schottky-barrier contact, carriers are injected directly from the metal into the empty space of semiconductor. So, current density $J_{s \rightarrow m}$ is defined [13] as

$$
J_{s \rightarrow m}=e \int_{-\infty}^{+\infty} v_{x} d n
$$

where $e$ is the magnitude of the electronic charge and $v_{x}$ is the carrier velocity in the direction of transport. Kinetic energy is utilized as a main parameter over the Fermi level to calculate the current density [14]. By substitution of the carrier concentration model in (7), the current of BGN schottky-barrier diode is analytically derived as (8). As specified in (8), the current of the proposed diode is a function of various physical and electrical characteristics including the carrier effective mass $\left(m^{*}\right)$, channel area $(A)$, temperature $(T)$, applied bias voltage $\left(V_{A}\right)$, and thermal voltage $\left(V_{T}\right)$ :

$$
\begin{aligned}
& I_{s \rightarrow m} \\
& =\frac{\sqrt{2} e\left(k_{B} T\right)^{3 / 2} A}{2 \pi \sqrt{m^{*}}} \\
& \times \int_{0}^{\eta}\left(x^{1 / 2} d x\right. \\
& \times\left(\left[4 \beta\left(\frac{\alpha \pm \sqrt{\alpha^{2}-4\left(k_{B} T\right) \beta} x}{2 \beta}\right)^{3 / 2}\right.\right. \\
& \left.-2 \alpha\left(\frac{\alpha \pm \sqrt{\alpha^{2}-4\left(k_{B} T\right) \beta x}}{2 \beta}\right)^{1 / 2}\right] \\
& \left.\left.\times\left(1+e^{x-\eta}\right)\right)^{-1}\right)
\end{aligned}
$$

where $A$ is the area of the channel which is proportional with channel width, $x=\left(E-E_{C}\right) / k_{B} T$, and $\eta \approx\left(V_{A}-V_{T}\right) /\left(k_{B} T / e\right)$ [31].

\section{Results and Discussion}

The purpose of this study is to highlight the influence of BGN geometry and temperature characteristics on the performance of the schottky-barrier diode. As can be seen in Figure 6, the rectification current-voltage characteristic of the proposed diode at different values of the width is illustrated. Apparently, there is a significant rise in the forward current of the diode as the BGN width increases. Strong dependence of I-V characteristic to the mentioned geometry parameter demonstrates that the increment of BGN width plays an important role in the forward current of the device. In other words, the diode performance will be enhanced by the leftshifted turn-on voltage. To get a better insight into the effect of BGN geometry on the increment of the diode current, two significant factors play an important role, which are the transparency of schottky-barrier contact and the extension of the energy for carrier concentration [32]. For the first parameter, as the diode current and schottky-barrier height are affected significantly by the charges, the channel width effect on the current through the schottky-barrier contact is taken into account in the proposed model. Furthermore, when the center of the channel is unoccupied with the charge impurities, the current increases due to the fact that free electrons are not affected by the positive charges [32]. The effect of second parameter emerges at the beginning of the channel where the barrier potential reduces as a result of 
TABLE 1: Turn-on voltage of BGN schottky-barrier diode at different values of BGN width.

\begin{tabular}{lccccc}
\hline BGN Width (nm) & 10 & 50 & 100 & 150 & 200 \\
Turn-on Voltage (V) & 0.95 & 0.92 & 0.88 & 0.87 & 0.86
\end{tabular}

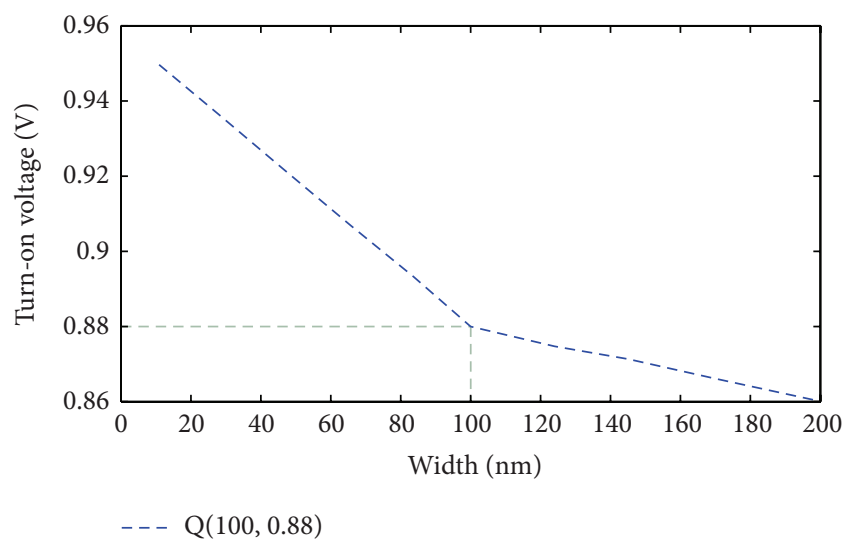

FIGURE 7: Turn-on voltage versus width characteristic of BGN schottky-barrier diode.

low charge density. This phenomenon leads to widening the energy casement and relieve of electron flow in the channel [32]. Moreover, due to the long mean free path (MFP) of GNR, scattering effect is not dominant [32]; therefore increasing the BGN geometry will result in a larger forward current.

Figure 7 indicates the turn-on voltage versus width characteristic of BGN schottky-barrier diode in which the turnon voltage of diode decreases by increasing the width of BGN. As depicted in Figure 7, Q $(100,0.88)$ as an inflection point can be considered a turning point after which a remarkable change is expected to result.

According to Figures 6 and 7, the turn-on voltage of the diode is obtained for different values of BGN width as shown in Table 1.

Thermodynamic stability is one of the most important properties of BGN [30]. In fact, the carrier transport in BGN is a vital phenomenon that determines the I-V characteristic of the device. The effect of temperature on current-voltage characteristic of BGN schottky-barrier diode is investigated in Figure 8. According to the relationship between the current and conductance [31], the presented model indicates a strong temperature dependence of current-voltage characteristic showing that the lower value of turn-on voltage appears as the more temperature increases.

In other words, the turn-on voltage will be shifted leftwards and the proposed diode performance will be enhanced. In fact, the conductance of BGN is expected to be affected by the temperature. It has been demonstrated that the minimum conductance of BGN depends on the bias voltage [30]. In $\mathrm{BGN}$, the temperature dependence of conductance is strongly affected by lower applied perpendicular electric field, and weakly temperature dependence of conductance has been

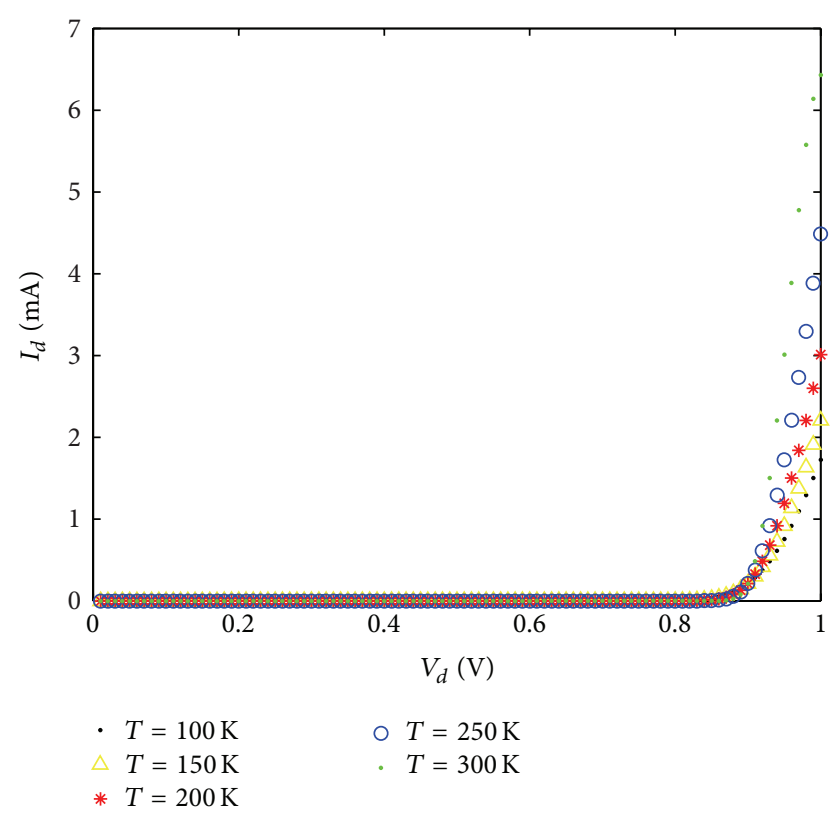

FIGURE 8: Rectification I-V characteristic of BGN schottky-barrier diode at different temperatures $(w=100 \mathrm{~nm}, l=100 \mathrm{~nm})$.

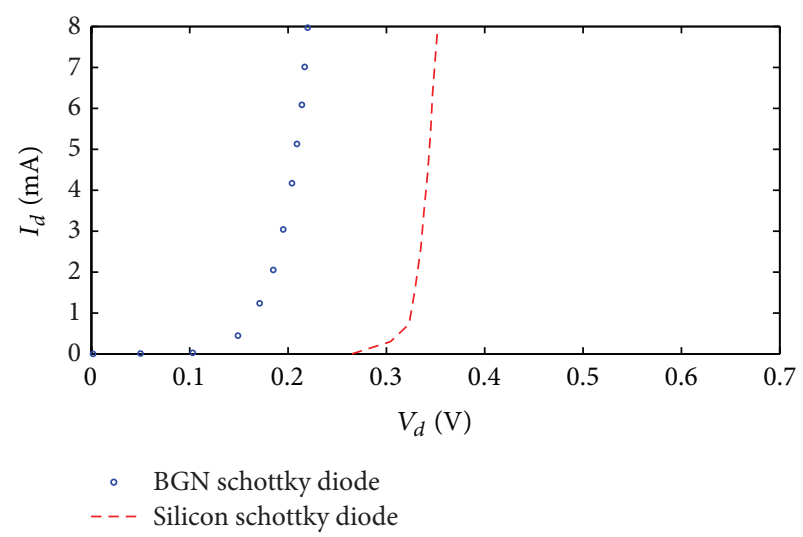

FIGURE 9: Comparison between BGN schottky-barrier diode with silicon schottky diode $[13,14]$.

reported in higher electric field [30]. According to the zerogap semiconductor characteristic, the conductance of GNR near the charge-neutrality peak is basically temperature independent for small bias voltages. In contrary, the temperature dependence of conductance in BGN is markedly different from that measured in the GNR. On the other hand, no sign of increase in BNG conductivity with increasing temperature away from the neutrality point has not been observed [30]. It is notable that the temperature dependence of currentvoltage characteristic in BGN revealed a new effect of a memory step close to the charge neutrality voltage. The effect is related to the slow relaxation processes in BGN. This characteristic of electron transport in BGN can be adopted in high-temperature applications. It is concluded 


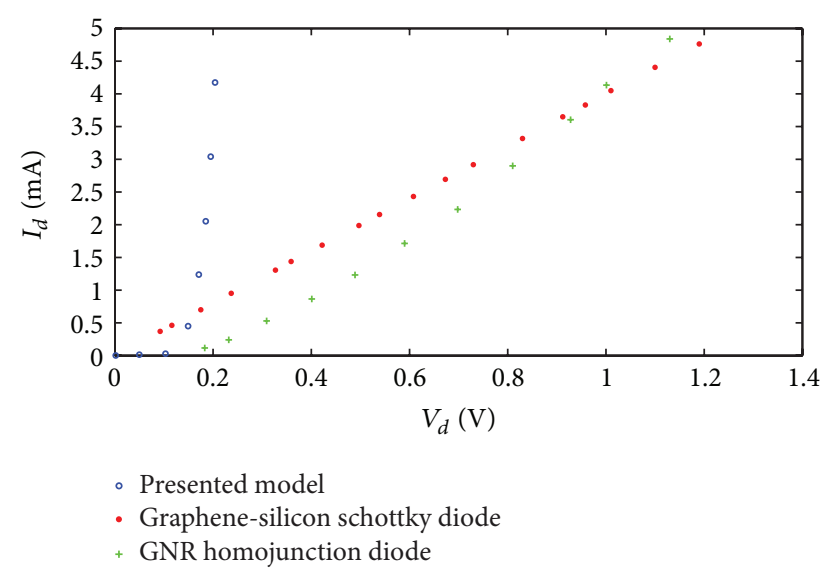

FIGURE 10: Comparison between the BGN schottky-barrier diode with graphene-silicon schottky diode and GNR homojunction diode $[6,15]$.

that temperature is an effective factor in the forward current and turn-on voltage of BGN schottky-barrier contact, leading to movement of free carriers in diode. According to the simulated result in Figure 7 , the point of $Q(100,0.88)$ is an inflection point, and hence the width of $100 \mathrm{~nm}$ is considered as an inflection width in Figure 8.

Figure 9 indicates a comparative study of the proposed device and the typical I-V characteristic of a silicon schottky diode $[13,14]$. Apparently, the effective turn-on voltage of silicon schottky diode is about $0.3 \mathrm{~V}$. The proposed BGN schottky-barrier diode also possesses a turn-on voltage of $0.1 \mathrm{~V}$ that is smaller than the voltage of silicon schottky diode. Accordingly, the proposed diode shows a better performance compared to the conventional silicon schottky diode in terms of the turn-on voltage.

As shown in Figure 10, the proposed BGN schottkybarrier diode has a better performance compared to the graphene-silicon schottky diode and GNR homojunction diode in terms of forward current. Moreover, smaller turnon voltage of the proposed diode $(0.1 \mathrm{~V})$ in comparison with GNR homojunction diode is indicated in Figure 10. Accordingly, an acceptable rectifying performance is seen comparable with conventional rectification behavior of the schottky diodes.

Difference between the BGN schottky-barrier diode and the conventional diodes in terms of mentioned electrical parameters can be associated with diode switching characteristics. It clearly gives an illustration of the fact that GNR-based device characterized by steep subthreshold slope displays a faster transient between on-off states [32]. In fact, a small value of subthreshold slope denotes a small change in the input bias which can modulate the output current and thus leads to less power consumption [32]. It is concluded that due to some excellent properties of GNR such as quantum transport, long spin-diffusion length, and extremely high carrier mobility [32], the proposed schottkybarrier diode can be used as a high speed device in future nanoelectronics.

\section{Defects Effect on the Device Performance}

There are various controllable defects in GNR, including the adatoms, vacancies, substitution, disorder, and stone wales (SW) defects [33]. It is noteworthy that the electronic properties of GNR can be functionalized via inhomogeneities, vacancies, topological defects, doping, adsorption, chemical functionalization, and molecular junctions [34]. It has been investigated that the existence of the defects in GNR is energetically more favorable than in fullerene or CNT [33]. The inhomogeneities, vacancies, and defects can lead to scattering in GNR. These defects persuade long-range deformations, which alter the electron routes [35]. The bond angle of the edge close to the SW defects is reduced from $120^{\circ}$ to $116^{\circ}$. In contrast to the shrinking through the width axis, the SW defects stretch from $4.88^{\circ} \mathrm{A}$ to $5.38^{\circ} \mathrm{A}$ through the length axis direction, and the transformation energy for the symmetric SW defects is $5.95 \mathrm{eV}$ [33]. SW defects have been predicted to modify the band structure and DOS of GNR and hence to impact upon its transport properties [36]. Recent results demonstrate that the defects alter GNR's chemical reactivity on the chemisorption processes [36]. SW defects can enhance the tendency of graphitic layers to transform into the nonplanar nanostructures, and the defects can play an important role in the intrinsic rumpling of GNR [37]. Additionally, the symmetry effects yield a remarkable conductance decrease in the SW defects configuration [33]. Moreover, the maximum value of Fermi velocity considering the SW defects is $5.25 \times 10^{5} \mathrm{~m} / \mathrm{s}$ which is about $50 \%$ less than the perfect GNR. This is because the defects modify the electron trajectories [38]. According to the ballistic conductance and carrier velocity dependence of current-voltage characteristic $[39,40]$, it is concluded that the performance of the schottkybarrier diode can be infused by inhomogeneities, vacancies, and defects. As a future work, the effects of SW defects on the BGN schottky-barrier diode performance will be analytically studied.

\section{Conclusion}

In this study, BGN with various stacking arrangements (AA and $\mathrm{AB}$ ) is applied as a metal and semiconductor contact in a junction schottky-barrier device. According to this assumption, an analytical model for BGN schottky-barrier diode is presented, and the effect of BGN geometry and temperature on I-V characteristic of the device is studied. Based on what has been discussed, by increasing the BGN width, the forward current of diode increases. Moreover, the simulated result indicates that the lower value of turn-on voltage appears as the more temperature increases, which guarantees a better performance of the proposed diode. Finally, a comparative study of the proposed model with silicon schottky diode, GNR homojunction contact, and graphene-silicon schottky diode is presented. Accordingly, an acceptable rectifying performance is seen comparable with conventional rectification behavior of the schottky diodes. To optimize the performance of BGN schottkybarrier diode, the presented model can be applied as a useful 
tool. It is concluded that the model can assist in comprehending experiments involving GNR schottky-barrier based devices.

\section{Acknowledgments}

The authors would like to acknowledge the financial support by Research University grant of the Ministtry of Higher Education (MOHE), Malaysia, under Projects Q.J130000.7123.02H24 and Q.J130000.7123.02H04. Also thanks are due to the Research Management Center (RMC) of Universiti Teknologi Malaysia (UTM) for providing excellent research environment in which they completed this work.

\section{References}

[1] K. S. Novoselov, A. K. Geim, S. V. Morozov et al., "Electric field in atomically thin carbon films," Science, vol. 306, no. 5696, pp. 666-669, 2004.

[2] A. Kargar and D. L. Wang, "Analytical modeling of graphene nanoribbon Schottky diodes," in Carbon Nanotubes, Graphene and Associated Devices III, vol. 7761 of Proceedings of the SPIE, San Diego, Calif, USA, August 2010.

[3] K. Alam, "Transport and performance of a zero-Schottky barrier and doped contacts graphene nanoribbon transistors," Semiconductor Science and Technology, vol. 24, no. 1, Article ID 015007, 2009.

[4] M. H. Ghadiry, M. Nadi, M. Rahmani, M. T. Ahmadi, and A. B. A. Manaf, "Modelling and simulation of saturation region in double gate graphene nanoribbon transistors," Semiconductors, vol. 46, no. 1, pp. 126-129, 2012.

[5] M. T. Ahmadi, Z. Johari, N. A. Amin, A. H. Fallahpour, and R. Ismail, "Graphene nanoribbon conductance model in parabolic band structure," Journal of Nanomaterials, vol. 2010, Article ID 753738, 4 pages, 2010.

[6] M. T. Ahmadi, M. Rahmani, M. H. Ghadiry, and R. Ismail, "Monolayer graphene nanoribbon homojunction characteristics," Science of Advanced Materials, vol. 4, no. 7, pp. 753-756, 2012.

[7] Y. Ouyang, Y. Yoon, and J. Guo, "Scaling behaviors of graphene nanoribbon FETs: a three-dimensional quantum simulation study," IEEE Transactions on Electron Devices, vol. 54, no. 9, pp. 2223-2231, 2007.

[8] Y. Yoon, G. Fiori, S. Hong, G. Iannaccone, and J. Guo, "Performance comparison of graphene nanoribbon FETs with Schottky contacts and doped reservoirs," IEEE Transactions on Electron Devices, vol. 55, no. 9, pp. 2314-2323, 2008.

[9] Q. Zhang, T. Fang, H. Xing, A. Seabaugh, and D. Jena, "Graphene nanoribbon tunnel transistors," IEEE Electron Device Letters, vol. 29, no. 12, pp. 1344-1346, 2008.

[10] A. Naeemi and J. D. Meindl, "Conductance modeling for graphene nanoribbon (GNR) interconnects," IEEE Electron Device Letters, vol. 28, no. 5, pp. 428-431, 2007.

[11] Q. Liang and J. Dong, "Superconducting switch made of graphene-nanoribbon junctions," Nanotechnology, vol. 19, no. 35, Article ID 355706, 2008.

[12] D. Jena, T. Fang, Q. Zhang, and H. Xing, "Zener tunneling in semiconducting nanotube and graphene nanoribbon $\mathrm{p}-\mathrm{n}$ junctions," Applied Physics Letters, vol. 93, no. 11, Article ID 112106, 3 pages, 2008.
[13] D. A. Neamen, Semiconductor Physics and Devices, University of New Mexico, Albuquerque, NM, USA, 3rd edition, 2003.

[14] M. Rahmani, M. T. Ahmadi, R. Ismail, and M. H. Ghadiry, "Performance of bilayer graphene nanoribbon Schottky diode in comparison with conventional diodes," Journal of Computational and Theoretical Nanoscience, vol. 10, no. 2, pp. 323-327, 2013.

[15] C.-C. Chen, M. Aykol, C.-C. Chang, A. F. J. Levi, and S. B. Cronin, "Graphene-silicon Schottky diodes," Nano Letters, vol. 11, no. 5, pp. 1863-1867, 2011.

[16] S. Sankaran and K. O. Kenneth, "Schottky barrier diodes for millimeter wave detection in a foundry CMOS process," IEEE Electron Device Letters, vol. 26, no. 7, pp. 492-494, 2005.

[17] A. Kargar and C. Lee, "Graphene nanoribbon schottky diodes using asymmetric contacts," in Proceedings of the 9th IEEE Conference on Nanotechnology, pp. 243-245, Genoa, Italy, July 2009.

[18] D. Jimenez, "A current-voltage model for Schottky-barrier graphene-based transistors," Nanotechnology, vol. 19, no. 34, Article ID 345204, 2008.

[19] S. M. Mousavi, M. T. Ahmadi, H. Sadeghi et al., "Bilayer graphene nanoribbon carrier statistic in degenerate and non degenerate limit," Journal of Computational and Theoretical Nanoscience, vol. 8, no. 10, pp. 2029-2032, 2011.

[20] S. Latil and L. Henrard, "Charge carriers in few-layer graphene films,” Physical Review Letters, vol. 97, no. 3, Article ID 036803, 4 pages, 2006.

[21] M. Koshino, "Electron delocalization in bilayer graphene induced by an electric field," Physical Review B, vol. 78, no. 15, Article ID 155411, 5 pages, 2008.

[22] M. Koshino, "Electronic transport in bilayer graphene," New Journal of Physics, vol. 11, no. 9, Article ID 095010, 2009.

[23] M. Rahmani, R. Ismail, M. T. Ahmadi, and M. H. Ghadiry, "Quantum confinement effect on trilayer graphene nanoribbon carrier concentration," Journal of Experimental Nanoscience, 2013.

[24] E. V. Castro, K. S. Novoselov, S. V. Morozov et al., "Electronic properties of a biased graphene bilayer," Journal of Physics Condensed Matter, vol. 22, no. 17, Article ID 175503, 2010.

[25] T. Stauber, N. M. R. Peres, F. Guinea, and A. H. C. Neto, "Fermi liquid theory of a Fermi ring," Physical Review B, vol. 75, no. 11, Article ID 115425, 10 pages, 2007.

[26] D. S. Novikov, "Numbers of donors and acceptors from transport measurements in graphene," Applied Physics Letters, vol. 91, no. 10, Article ID 102102, 2007.

[27] Z. Q. Li, E. A. Henriksen, Z. Jiang et al., "Band structure asymmetry of bilayer graphene revealed by infrared spectroscopy," Physical Review Letters, vol. 102, no. 3, Article ID 037403, 4 pages, 2009.

[28] E. V. Castro, N. M. R. Peres, J. M. B. L. Dos Santos, F. Guinea, and A. H. C. Neto, "Bilayer graphene: gap tunability and edge properties," Journal of Physics: Conference Series, vol. 129, no. 1, Article ID 012002, 2008.

[29] F. Guinea, A. H. C. Neto, and N. M. R. Peres, "Interaction effects in single layer and multi-layer graphene," European Physical Journal, vol. 148, no. 1, pp. 117-125, 2007.

[30] H. Sadeghi, S. M. Mousavi, M. Rahmani, M. T. Ahmadi, and R. Ismail, "Bilayer graphene nanoribbon transport model," in Advanced Nanoelectronics, chapter 7, Taylor \& Francis, New York, NY, USA, 2012. 
[31] S. Datta, Quantum Transport: Atom to Transistor, Cambridge University Press, New York, NY, USA, 2005.

[32] M. Rahmani, M. T. Ahmadi, H. Karimi, M. Saeidmanesh, E. Akbari, and R. Ismail, "Analytical modeling of trilayer graphene nanoribbon Schottky-barrier FET for high-speed switching applications," Nanoscale Research Letters, vol. 8, no. 1, article 55, 2013.

[33] H. Zeng, J. Zhao, J. W. Wei, and H. F. Hu, "Effect of N doping and Stone-Wales defects on the electronic properties of graphene nanoribbons," The European Physical Journal B, vol. 79, no. 3, pp. 335-340, 2011.

[34] J. Kotakoski, A. V. Krasheninnikov, U. Kaiser, and J. C. Meyer, "From point defects in graphene to two-dimensional amorphous carbon," Physical Review Letters, vol. 106, no. 10, Article ID 105505, 4 pages, 2011.

[35] A. H. C. Neto, F. Guinea, N. M. R. Peres, K. S. Novoselov, and A. K. Geim, "The electronic properties of graphene," Reviews of Modern Physics, vol. 81, no. 1, pp. 109-162, 2009.

[36] L. Chen, H. Hu, Y. Ouyang, H. Z. Pan, Y. Y. Sun, and F. Liu, "Atomic chemisorption on graphene with Stone-Thrower-Wales defects," Carbon, vol. 49, no. 10, pp. 3356-3361, 2011.

[37] J. Ma, D. Alfè, A. Michaelides, and E. Wang, "Stone-Wales defects in graphene and other planar $s p^{2}$-bonded materials," Physical Review B, vol. 80, no. 3, Article ID 033407, 4 pages, 2009.

[38] Y. J. Sun, F. Ma, D. Y. Ma, K. W. Xu, and P. K. Chu, "Stressinduced annihilation of Stone-Wales defects in graphene nanoribbons," Journal of Physics D, vol. 45, no. 30, Article ID 305308, 2012.

[39] M. Rahmani, R. Ismail, M. T. Ahmadi, M. J. Kiani, and K. Rahmani, "Carrier velocity in high-field transport of trilayer graphene nanoribbon field effect transistor," Science of Advanced Materials. In press.

[40] M. Rahmani, M. T. Ahmadi, H. F. A. Karimi, M. J. kiani, E. Akbari, and R. Ismail, "Analytical modeling of monolayer graphene-based $\mathrm{NO}_{2}$ sensor," Sensor Letters, vol. 11, no. 2, pp. 270-275, 2013. 

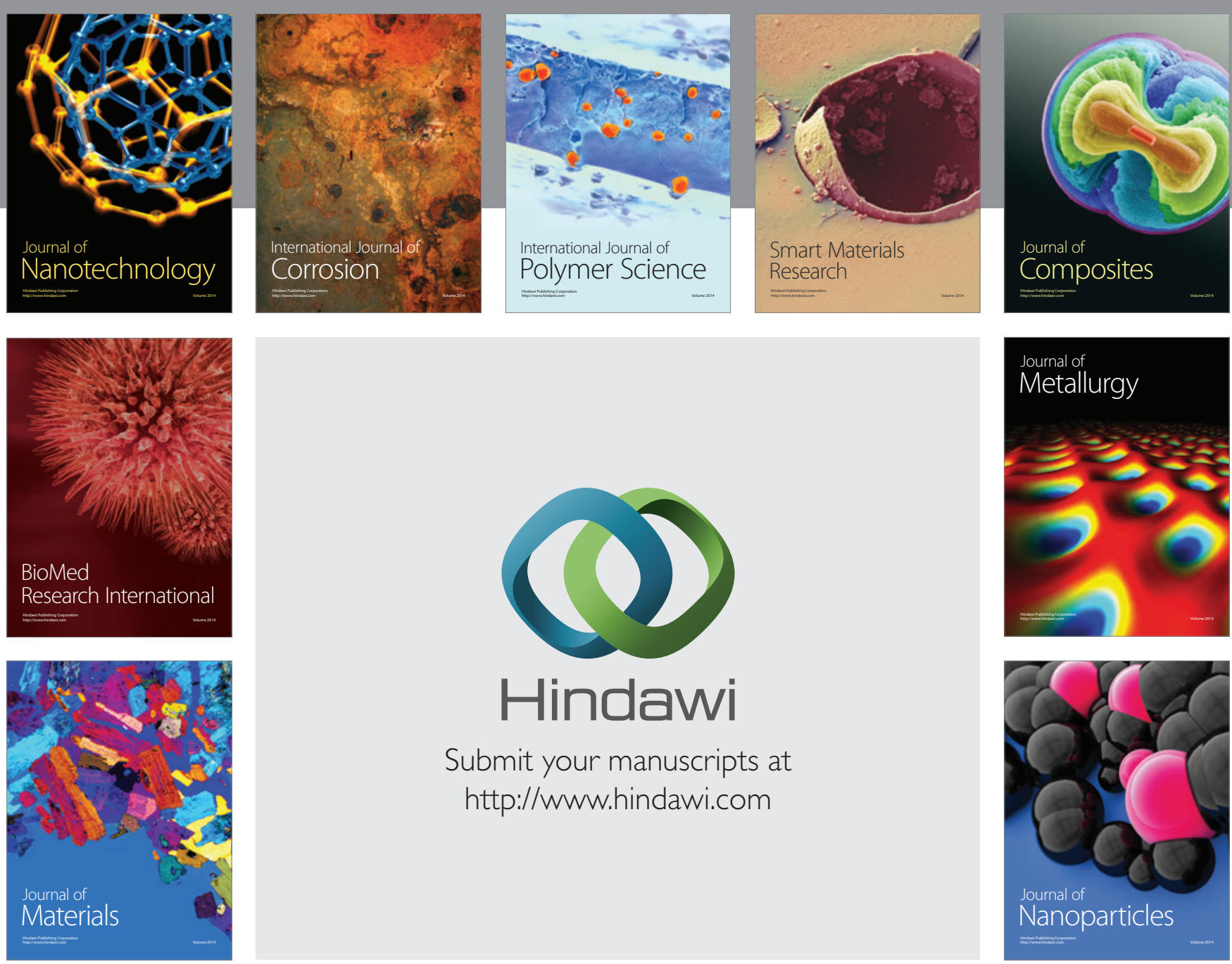

Submit your manuscripts at http://www.hindawi.com
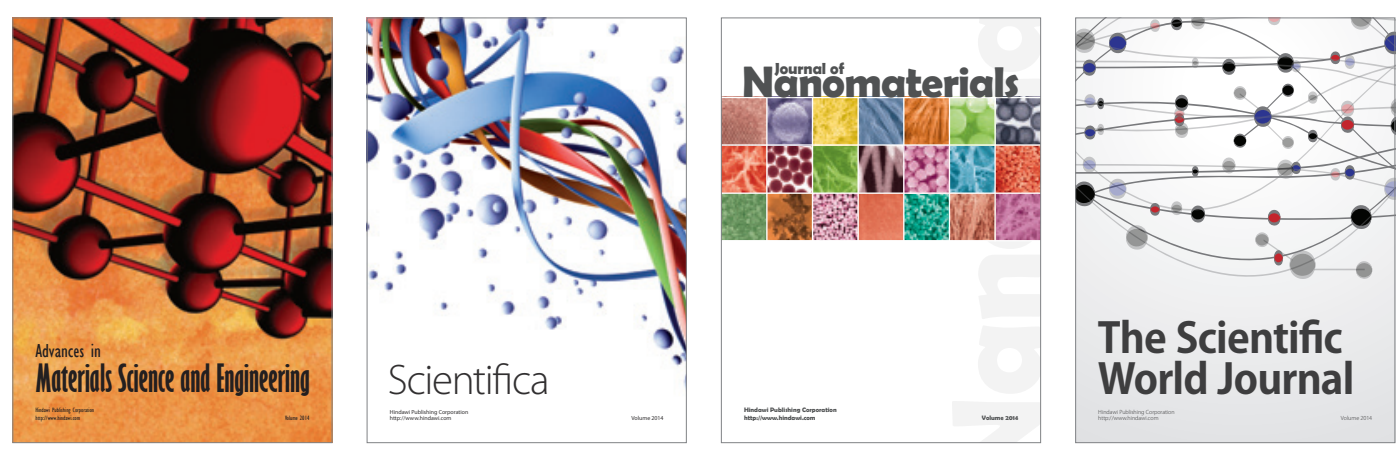

\section{The Scientific World Journal}
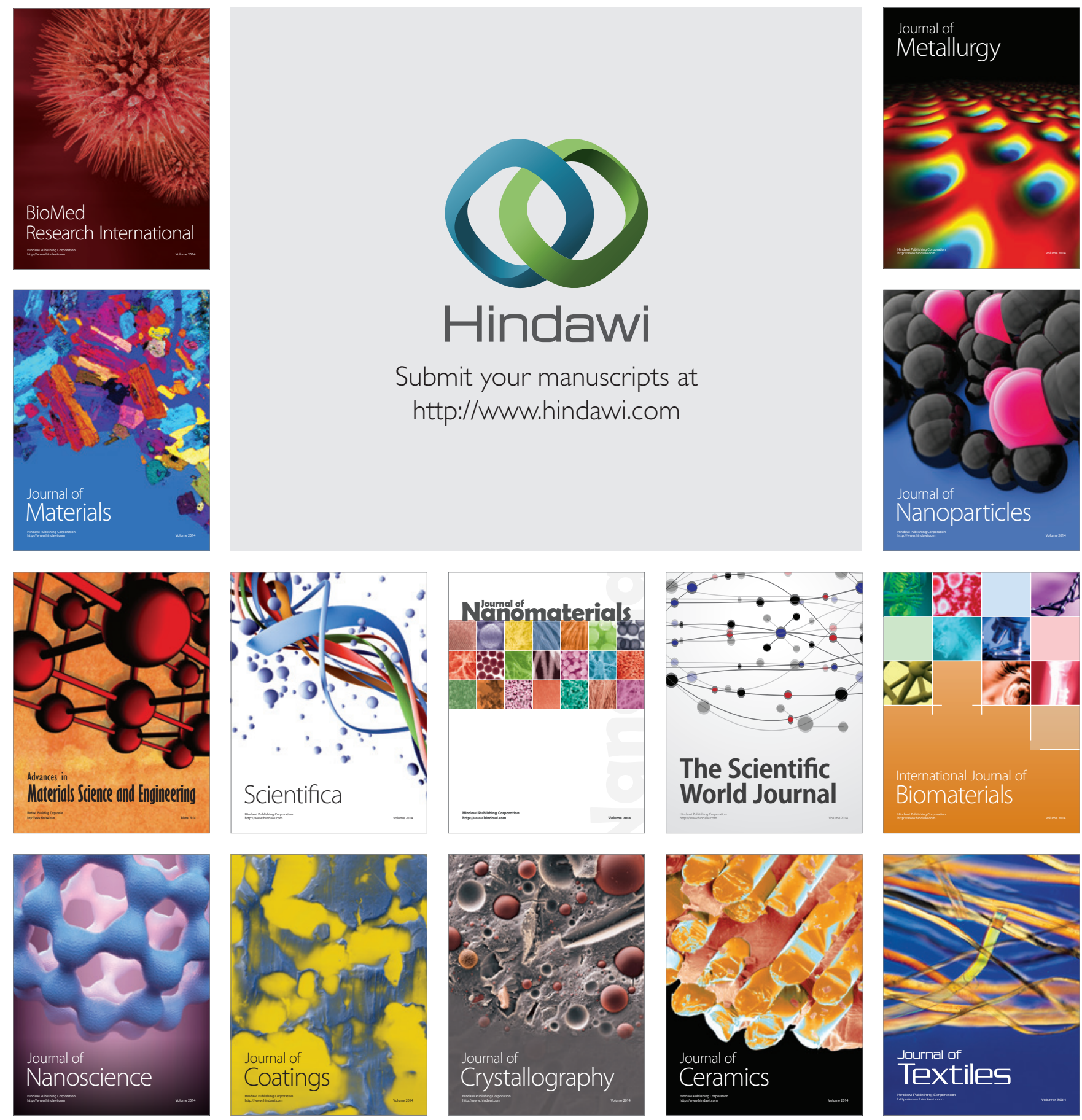